\title{
PATIENT-INDIVIDUAL HIP CUPS: SIMULATION-BASED DESIGN AND SHEET METAL FORMING MANUFACTURING
}

\author{
Betancur Escobar, S. ${ }^{1}$, Bouguecha, A. ${ }^{1}$, Almohallami, A. ${ }^{1}$, Niemeier, H. ${ }^{1}$, Nolte, I. ${ }^{2}$, Lucas, K. ${ }^{2}$, \\ Stukenborg-Colsmann, C. ${ }^{3}$, Lerch, M. ${ }^{3}$, Behrens, B.-A. ${ }^{1}$ \\ ${ }^{1}$ Institute of Forming Technology and Machines, Leibniz Universität Hannover,Germany \\ ${ }^{2}$ Department of Orthopaedic Surgery, Hannover Medical School, Germany \\ ${ }^{3}$ Small Animal Clinic, University of Veterinary Medicine Hannover, Germany \\ betancur@ifum.uni-hannover.de
}

\begin{abstract}
The revision of an hip prosthesis can have diffrent reasons. One frequent cause, especialley after implantation of a conventional cup, is the so called stress-shielding effect which can lead to a migration or loosening. Patientspecific hip cups can be used to counteract this. However, individual hip cups are only implanted for the treatment of great deformations or tumours because of the cost-intensive manufacturing. Within this project a patient-specific hip cup prosthesis has to be developed and manufactured. Besides the numerical design by means of a coupling between multi-body simulation (MBS) and finite element method (FEM), an inovative concept for the production of patientindividual hip prosthesis out of titanium sheets is introduced in this study.
\end{abstract}

Patient-individual hip cups, bone remodelling, reverse engineering, process planning, sheet metal forming

\section{Introduction}

The long-term result of hip prostheses is mainly determined by migration or aseptic loosening caused by bone remodelling. Especially the migration of the artificial hip cup is a major problem [1][2]. The migration is a concequence from the changed mechanical conditions. This change can lead to a bone remodelling caused by stress shieding [3]. In addition, for a sufficiently stable anchoring of the hip cup a high bone resection is necessary which promotes the bone resorption and complicates a revision surgery.Patient-specific hip cups can be used to counteract these problems. However, individual hip cups are only implanted for the treatment of great deformations or tumours in the hip joint due to the time consuming and cost-intensive manufacturing [4]. Within this project a concept for the economical production of human and canine patient-specific hip cup prosthesis has to be established. Besides the numerical design by means of a coupling between multi-body simulation (MBS) and finite element method (FEM), an inovative concept for the production of patient-individual hip prosthesis out of titanium sheets is introduced in this study.

\section{Numerical Design}

In a prior study the bone remodelling after implantation of a conventional hip cup was calculated (Fig. 1). Therefore a coupled simulation by means of a MBS and FEM was used. The numerical results show a high bone resorption in the acetabulum. A migration of the conventional cup can be suggested in the proximal direction, beacause of the final density distribution. For patient-individual hip cups bone resorption is not assumed to appear in that extent. First calculations indicate a less severe bone resorption with patient specific prostheses. This is due to the near physiological design of patientindividual prostheses. Thus, less bone resorption can be expected.

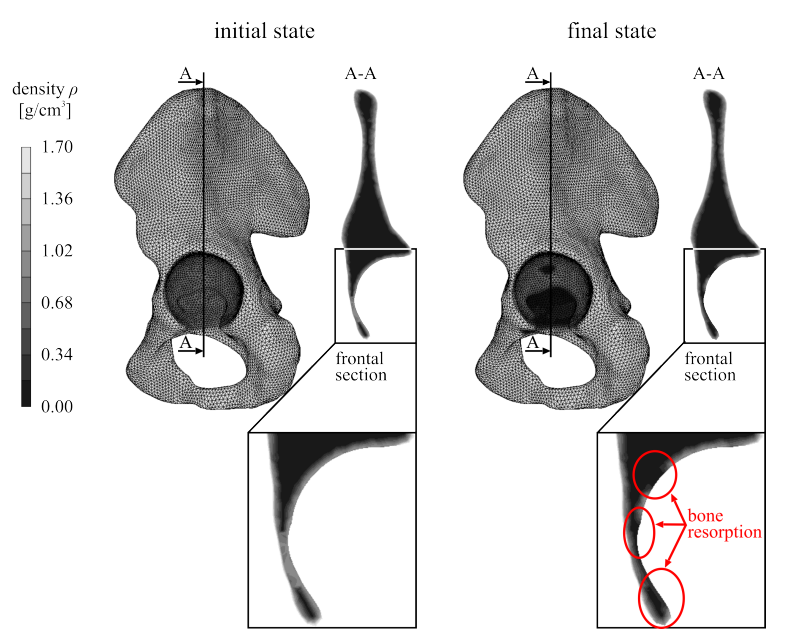

Figure 1: Density distributions in the periprosthetic pelvis for the initial and final state [5]

\section{Production Concept}

The concept for the production of the patient-indivdual hip cup is devided in two process steps. First standardized titanium sheet metal components have to be produced, then a true-size enlargement of these components according to the patient-specific geometries of the acetabula is to be executed.

Whereas the true-size entlagement will be carried out by a double acting rubber-die forming, two diffrent processes be worth considering for the manufacturing of the standardized components: punch-die way of forming (with punch, blank holder and die) and high pressure sheet metal forming (HPF). Due to the results of the process simulation via FEM, the HPF process at room temperature will be 
choosed for the first production step. In this process the titanium blank is pressed against a die that has the shape of a canine universal acetabulum geometry using a pressing medium like oil.
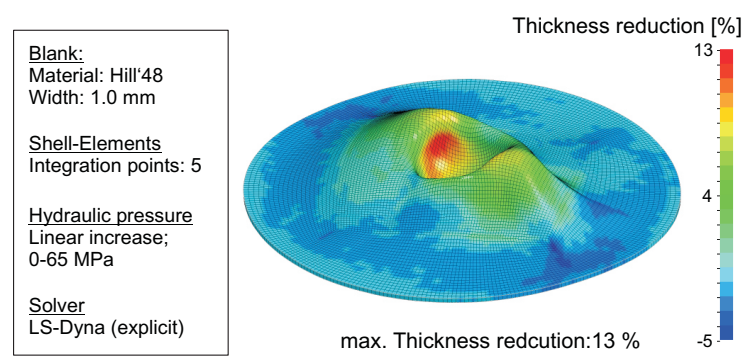

Figure 2: Results of the process simulation

According to the results (Fig. 2) the HPF-procces would cause reducing in the blank thickness by about $13 \%$, which can be considered as quite safe value to go over to the real process design. In contrast, the reduction of the blank thickness afer the punch die way of forming is about $29 \%$, which is rated as critical.

\section{Design Method}

The canine universal acetabulum geometry used for the simulations, results from a design method developed in this project with a dataset of 15 creossbreed dogs. In Fig. 3 the design chain of this method is illustrated. First CT-data are collected and then segmented so that only the geometry of the pelvis is present as 3D-model. After that the resulting 3D-models of the pelvis are trimmed reproducible. Thus only the edge of the acetabulum remains for the creation of the universal geoemtry.

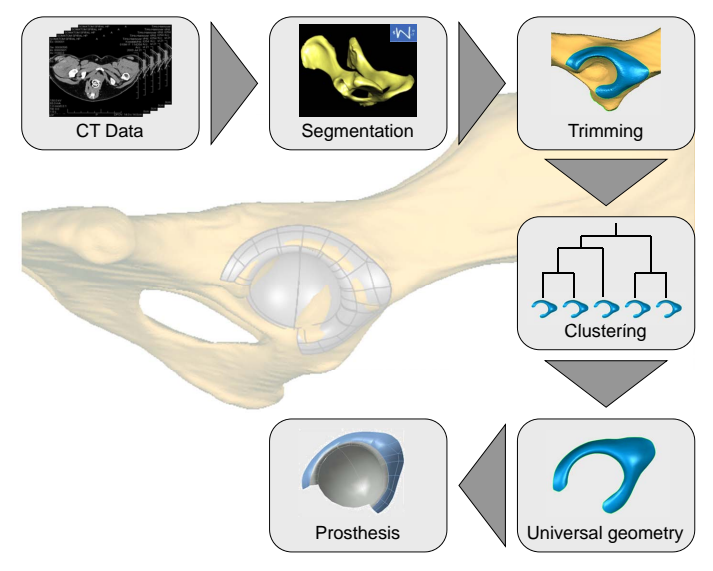

Figure 3: Generation of a mean geoemtry

With these trimmed geoemtries the deduction of the universal geomtry is carried out. For this the hierarchical agglomerative clustering is used. Thereby always two geometries are combined to a mean geometry which have the lowest dimensional deviation. This combinaiton method is repeated until one geomtry remains: the univerversal geometry. With this universal geomtery of the edge of the acetabulum a idealized hip cup prosthesis is designed.

\section{Outlook}

The next step of this project will be the design and test of the HPF die for the production of the universal geometry. For this the contour of the first generated canine universal geometry will be used. Furthermore the results from the numerical design will influence the conception of the new tool system to be developed.

\section{Acknowledgement}

The study is based on the framework of the Collaborative Research Center 599 "Sustainable degradable and permanent implants out of metallic and ceramic materials" and is a part of the subprojects D6 and D13. The authors would like to thank the German Research Foundation (DFG) for the financial support.

\section{Bibliography}

[1] E. Garcia-Cimbrelo, A. Diaz-Martin, R. Madero, and L. Munera, "Loosening of the cup after low-friction arthroplasty in patients with acetabular protrusion. the importance of the position of the cup," The Journal of bone and joint surgery. British volume, vol. 82, no. 1, pp. 108-115, 2000.

[2] M. B. Laursen, P. T. Nielsen, and K. Søballe, "Bone remodelling around ha-coated acetabular cups: A dexa study with a 3-year follow-up in a randomised trial," International orthopaedics, vol. 31, no. 2, pp. 199-204, 2007.

[3] B.-A. Behrens, I. Nolte, P. Wefstaedt, C. StukenborgColsman, and A. Bouguecha, "Numerical investigations on the strain-adaptive bone remodelling in the periprosthetic femur: influence of the boundary conditions," Biomedical engineering online, vol. 8, p. 7, 2009.

[4] M. F. Zäh, Wirtschaftliche Fertigung mit RapidTechnologien: Anwender-Leitfaden zur Auswahl geeigneter Verfahren. Kostengünstig Produzieren, München: Hanser, 2006.

[5] A. Bouguecha, N. Weigel, S. Escobar, I. Nolte, P. Wefstaedt, C. Stukenborg-Colsman, and B.-A. Behrens, "Influence of assumed boundary conditions derived from mbs on numerically simulated strain-adaptive bone remodeling in the pelvis after total hip replacement," in 5th European Conference of the International Federation for Medical and Biological Engineering (Á. Jobbágy, ed.), vol. 37 of IFMBE Proceedings, pp. 339-342, Springer Berlin Heidelberg, 2012. 